REVIEW ARTICLE

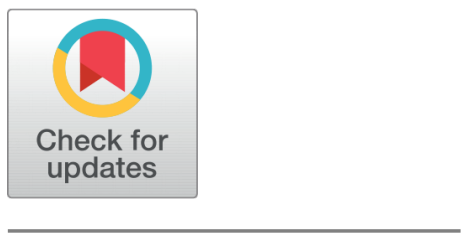

OPEN ACCESS

Received: 22-04-2020

Accepted: $10-08-2020$

Published: 19-08-2020

Editor: Dr. Natarajan Gajendran

Citation: Bagri M, Kumar S (2020) Solitons transmission system : A dynamic shift in optical fiber communication. Indian Journal of Science and Technology 13(30): 3093-3102. https://doi.org/ 10.17485/IJST/v13i30.384

* Corresponding author.

Tel: +91-9468403060

Manjeetbagri27@gmail.com

Funding: None

Competing Interests: None

Copyright: (c) 2020 Bagri \& Kumar. This is an open access article distributed under the terms of the Creative Commons Attribution License, which permits unrestricted use, distribution, and reproduction in any medium, provided the original author and source are credited.

Published By Indian Society for Education and Environment (iSee)

ISSN

Print: 0974-6846

Electronic: 0974-5645

\section{Solitons transmission system : A dynamic shift in optical fiber communication}

\author{
Manjeet Bagri ${ }^{1 *}$, Suresh Kumar ${ }^{2}$ \\ 1 M. Tech. student, Department of Electronics and Communication, Maharshi Dayanand \\ University, 124001, Haryana, India. Tel.: +91-9468403060 \\ 2 Assistant Professor, Department of Electronics and Communication, Maharshi Dayanand \\ University, 124001, Haryana, India
}

\section{Abstract}

Objective: To present a detailed study of Solitons transmission system, including their types, applications, effects, and updated research work and research gaps. Findings: The $4^{\text {th }}$, generation fiber optic communication system utilizes visual amplification to decrease the demand for repeaters and WDM to increase the information power. Similarly, $5^{\text {th }}$ the phase of Optical Fiber Communication is now expending the distances range which the wavelength division multiplexing system will control. The traditional wave length frame called the $C$ ring covers the distance range 1.53 to 1.57 um and dry material has the low loss window promising extension of range to 1.30 to $1.65 \mathrm{um}$. Additional development includes the idea of visual solitons pulses that maintain their body by counteracting the results of distribution with nonlinear effects of this material by applying pulses of specific shape. Application: This will motivate the researchers to undertake research work in the field of Solitons communication to achieve improved design characteristics, reducing number of repeaters, cost and higher data rate transmissions.

Keywords: Inter Symbol Interference (ISI); Group Velocity Dispersion (GVD); Self-Phase Modulation (SPM); WDM; OFDM

\section{Introduction}

Solitons are high power and very narrow pulses; these are so stable that the size and shapes of pulses are maintained even when these are travelling with in any medium. They propagate at a constant velocity and don't spread in optical fiber even after a thousand of kilometers. The nonlinear and spreading effect in the soliton medium is caused due to cancellations. Optical pulses propagating along the fiber optics experiences GVD and SPM. GVD is a linear effect in which the refractive index varies with frequency. These different frequency component travels with different velocities. SPM results in variation in refractive index due to intensity. If the refractive index increases with increase in intensity or lead to time dependent phase change then SPM is called positive. Since phase derivative time related to frequency and SPM effect produces the change of movement spectrum with the combination of both positive GVD and SPM broadening of the pulse and with (-ve) GVD and (+ve) SPM propagate pulse with no distortion. A separate group of solitons model has been studies in different situations 
and in different contextual terms and is still continuing as the area of intensive research. Figure $1^{(1,2)}$ shows the formation of soliton.

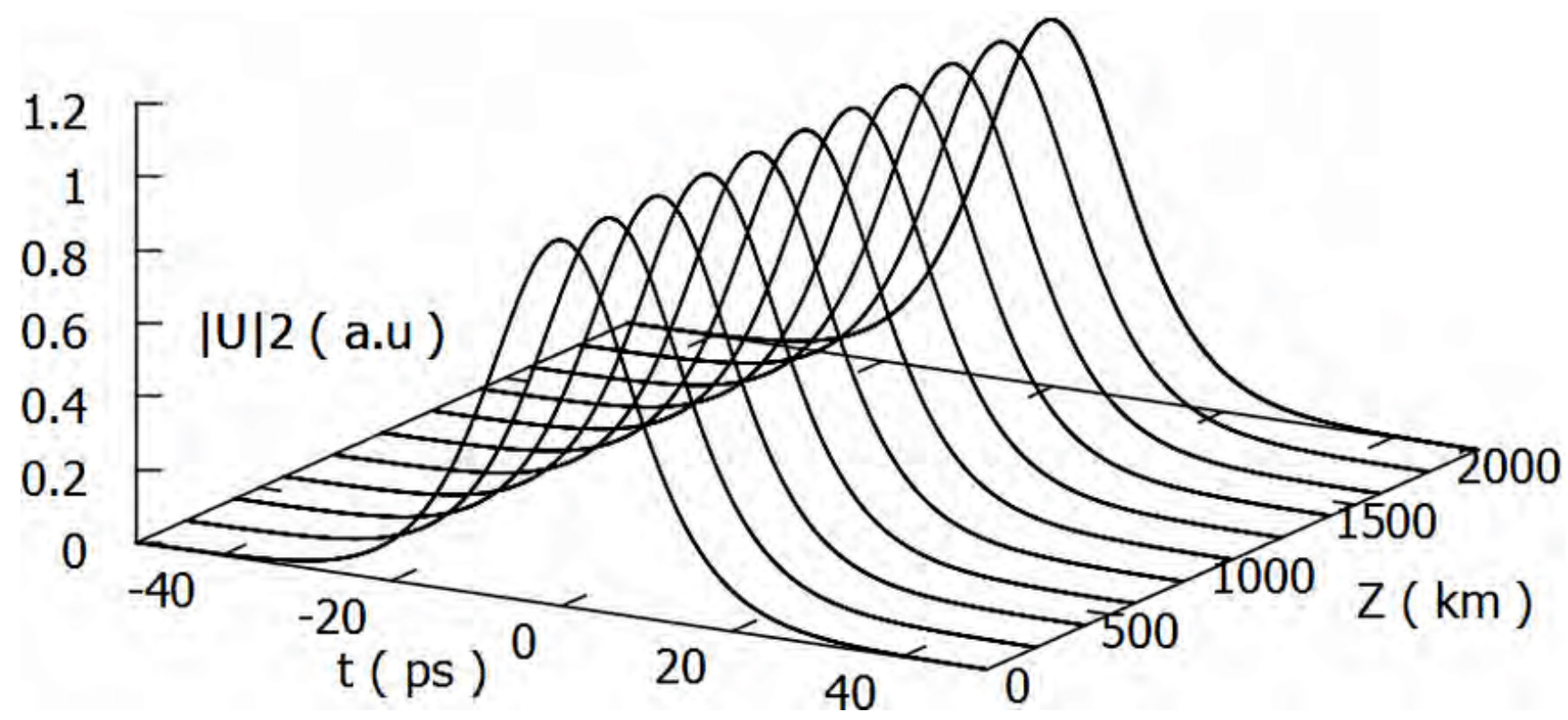

Fig 1. Basic Soliton formation

The study has been organized as, solitons possessing equations are given in section 2 followed by A detailed study of Type of Solitons in in Section 3. Section 4 explains the various advantages of optical solitons. Section 5 briefly summarizes the applications of optical Solitons followed by conclusion in section 6 .

\section{Solitons possessing equations}

Soliton and Rogue waves (RWs) are both are known as extreme waves which have received much interest recently in different area of physics. Fiber laser and fiber communication in different systems has remained a hot research topic. Fiber optics provide an ideal platform to observe the generation of optical RW and soliton as well as investigate their behaviour ${ }^{(3)}$.

Soliton assumes a significant job in fiber optics laser innovation. Bound states of solitons, also known as soliton molecules, are referred to that two or more fundamental solitons bind tightly together in the temporal or spatial domain through the direct soliton interaction. The solitons in the state have not only fixed separations but also fixed phase differences, and the assembly of the solitons behaves like a new super-soliton. Theoretically, the formation of bound states of solitons in the extended nonlinear Schrödinger equation (NLSE) systems were first predicted by B. Malomed. N. N. Akhmediev had also studied the formation of bound states of solitons in the complex Ginzburg-Landau equation (CGLE) systems. It was demonstrated that dissipative property delivers the solitons with one of a kind new highlights of communication that can bring about the arrangement of stable bound conditions of dissipative solitons ${ }^{(4,5)}$.

However, its nonlinear optical property and the related photonics device studied so far. Another method to generate soliton is bismuthene nanosheets and phosphorus that are synthesized by the sonochemical exfoliation method and transferred onto the taper region of a microfiber by the optical deposition method. Stable soliton pulses centered at $1561 \mathrm{~nm}$ with the shortest pulse duration of about $193 \mathrm{fs}$, repetition rate of $8.85 \mathrm{MHz}$ and average output power of $5.6 \mathrm{~mW}$, respectively. These are the shortest soliton pulses so far generated from a mode-locked laser based on the group VS 2D materials SAs ${ }^{(6,7)}$.

A solitary dissipative soliton can keep up to $152 \mathrm{mw}$, an exceptionally similarly sep rated uniform pulse train which show the solidness of the fiber laser. A high vitality and tunable dissipative solitons and wide range is extremely alluring to frequency division multiplexing applications. Manufactured polymethyl methacrylate PMMA-TIPMMA as saturable safeguard, which could bear long haul mechanical loadings, into the fiber laser hole created the stable dissipative soliton mode-locking with a 3 - $\mathrm{dB}$ ghastly data transfer capacity up to $51.62 \mathrm{~nm}$ and tunable wavelength range of $22 \mathrm{~nm}^{(8)}$.

Here we will discuss some of the important solitons including universal nonlinear equations 


\subsection{Korteweg and de Vries equation}

In 1985 Korteweg and De-Vries analyzed theoretically for the first time about wave train noticed by scott Russell later as KVD by the principle of dynamics. KVD equation is considered as the simple form of nonlinear ${ }^{(9)}$

$$
\frac{\partial q}{\partial t}+\frac{\partial^{3} q}{\partial x^{3}}+6 q \frac{\partial q}{\partial x}=0
$$

Where $\mathrm{q}(\mathrm{x}, \mathrm{t})$ is gradually shifting envelop, $x$ and $t$ are space coordinate and time respectively.

\subsection{Sine-Gordon equation}

In 1939 Frenkel and Kontorova presented an analysis type of problem in term of solid-state physic to maintain the relation between crystal disorder and plastic deformation. The motion of dislocation in the substances can be represented as

$$
\frac{\partial^{2} q}{\partial t^{2}}-\frac{\partial^{2} q}{\partial x^{2}}+\sin q=0
$$

Where $\mathrm{q}(\mathrm{x}, \mathrm{t})$ is gradually shifting envelope, $x$ and $t$ are space coordinate and time respectively.

\subsection{Toda lattice equation}

Toda lattice is named after Morikazu Toda who discovered in 1967. It leads with one dimensional array of homogenous mass fused using exponential type of nonlinear forces described as:

$$
\frac{d^{2} q_{n}}{d t^{2}}=\exp \left[-\left(q_{n}-q_{n-1}\right)\right]-\exp \left[-\left(q_{n+1}-q_{n}\right)\right]
$$

Where, $q n(t)$ is the longitudinal displacement of the $n$ atom from its equilibrium position.

\subsection{Non-linear Schrodinger equation}

The NLS equations describe the intensive light transmission in optical fiber ${ }^{(10)}$.

$$
i \frac{\partial q}{\partial t} \pm \frac{\partial^{2} q}{\partial x^{2}}+2|q|^{2} q=0
$$

Where $q(x, t)$ is gradually shifting envelope, $x$ and $t$ are space and time coordinates, + and - signs represent anomalous and normal dispersion respectively, the second and third term signifies the GVD and SPM of the system

$$
\begin{gathered}
\nabla . \nabla \psi=i \frac{\partial \psi}{\partial z}+\frac{\partial^{2} \psi}{\partial y^{2}}+\frac{\partial^{2} \psi}{\partial z^{2}}=\frac{1}{r^{2} \sin \theta}\left[\sin \theta \frac{\partial}{\partial r}\left(r^{2} \frac{\partial \psi}{\partial r}\right)+\frac{\partial}{\partial \theta}\left(\sin \theta \frac{\partial}{\partial \theta}\right)+\frac{1}{\sin \theta} \frac{\partial^{2} \psi}{\partial \varphi^{2}}\right] \\
i \frac{\partial \psi}{\partial z}=\alpha \frac{\partial^{2} \psi}{\partial \tau^{2}}+i \sigma \frac{\partial^{3} \psi}{\partial \tau^{3}}-\in \frac{\partial^{4} \psi}{\partial \tau^{4}}-\gamma|\psi|^{2} \psi-i \frac{\mu}{2} \psi
\end{gathered}
$$

Where, $\tau=t-\beta_{1} z$ is the retarded time, $\alpha=\alpha=\frac{\beta_{2}}{2}, \sigma=\frac{\beta_{3}}{6}, \in=\frac{\beta_{4}}{24}$, and $\gamma$ is the nonlinear parameter. The partameter $\beta_{k}=\left(d^{k} \beta / d \omega^{k}\right) \omega=\omega_{0}$

Where, $\beta$ and $\mathrm{k}$ are propagation constant and dispersion of optical fiber, On the basis of sign of last parameter $\mu$ in NLSE describe the absorption and amplification. Exact solitary wave solution for $\mu=0$

$\psi(z, \tau)=\operatorname{usech}^{2}\left[w\left(\tau-\eta-v^{-1} z\right) \exp [i(K Z-\delta \tau+\varnothing)]\right.$, Where $\eta$ and $\varnothing$ position and phase of pulse at $z=0$.

Amplitude and inverse temporal solitary wave are given by ${ }^{(11)}$

$$
\frac{\sqrt{-3}}{10 \gamma \in}\left(\frac{3 \sigma^{2}}{8 \varepsilon}-\alpha\right), w=\frac{1}{4} \sqrt{\frac{4 \alpha}{5 \epsilon}-\frac{3 \sigma^{2}}{10 \varepsilon^{2}}}
$$


Where, $\alpha<0, \in<0$ and $8 \alpha \in>3 \sigma^{2}$ with $\gamma>0$. In retarded frame velocity $v$ and parameters $\delta$ and $\kappa$ of solitary wave given as:

$$
\begin{gathered}
v=\frac{8 \varepsilon^{2}}{\sigma\left(\sigma^{2}-4 \alpha \varepsilon\right)}, \quad \delta=-\frac{\sigma}{4 \varepsilon}, \\
K=-\frac{4}{25 \varepsilon^{3}}\left(\frac{3 \sigma^{2}}{8}-\alpha \varepsilon\right)^{2}-\frac{\sigma^{2}}{16 \varepsilon^{3}}\left(\frac{3 \sigma^{2}}{16}-\alpha \varepsilon\right)
\end{gathered}
$$

Retarded time shows that $K+\beta_{1} \delta$ and $\delta$ are the wave number and frequency shifts. We insist that solitonsdoes not have nontrivial parameters. Velocities of these solitons are set as universal NLSE remain unchanged w. r. t. Galilean transformation $^{(12)}, \gamma>0$ yields with the next relations $\varepsilon<0 \alpha<3 \sigma^{2} / 8 \varepsilon$ In this case when $\beta_{3}>0$ optical solitons reduces for $\mu=0$ can also written as:

$$
i \frac{\partial \psi}{\partial z}=-\frac{\delta H}{\delta \psi^{*}}
$$

Where, Hrepresents Hamiltonian of the system

Development method proves the strength of the solitons. This method produce strength area similar to region of existence of sech $^{2}$ solitons: $\beta_{2}<0, \beta_{4}<0,2 \beta_{2} \beta_{4}>\beta_{3}^{2}$, where, $\beta_{3}$ may be negative positive or zero. Clearly, solitons solution and its energy is given by the Hamiltonian of roundedness. Energy of solitons is defined as:

$$
E=\int_{-\infty}^{+\infty}|\Psi(Z, \tau)|^{2} d \tau=\frac{4}{\gamma \sqrt{5|\varepsilon|}}\left(\frac{3 \sigma^{2}}{8 \varepsilon}-\alpha\right)^{3 / 2} 4
$$

Energy E and the solitons parameters fill simple scaling relations and the dispersion is denoted by $\beta_{4}=\beta_{k}^{(0)} q$ where, $k i s 2-4$ and $\mathrm{q}$ gives +ve dimensionless parameters. The scaling relations are:

$$
E=E_{0} q, u=u_{0} q^{1 / 2}, v=v_{0} q^{-1}, K=k_{0} q
$$

And we have $\omega=\omega_{0}$ and $\delta=\delta_{0}$. Here $E_{0}$ is given with the changes in $\alpha \longmapsto \alpha_{0}, \sigma \longmapsto \sigma_{0}$, and $\varepsilon \longmapsto \varepsilon_{0}$ where $\alpha_{0}=\frac{\beta_{2}^{(0)}}{2}, \sigma_{0}=$ $\frac{\beta_{3}^{(0)}}{6}$, and $\varepsilon_{0}=\frac{\beta_{4}^{(0)}}{24}$

Same changes are assumed for all other relations, If the $\mathrm{q} \alpha \mathrm{E}$, the value $|v|^{-1}$ increases proportional to parameter $\mathrm{q}$. In such situation the amplitude of the solitons pulse increases as $q^{1 / 2}$. Although $w^{-1}$ and the $\delta$ remain same as the parameter q grows.

Velocity of the solitons is given by $v_{s}=v /\left(1+\beta_{1} v\right)$ Hence, solution's velocity tends to zero

when $q \rightarrow \infty$ because it results the scaling relation

$$
v_{s}=\frac{v_{0}}{\left(q+\beta_{1} v_{0}\right)}
$$

Parameter $\mathrm{q}$ have some limits in optical fiber, we have determined that building a new kind of solitons pulse with low speed and high energy an appropriate dispersion profile is possible. Hence the scaling feature $s e c h^{2}$ of solitons can find various practical applications. All optical delay system dynamically manipulating the velocity of light has paid a great consideration to optical signal processing application that is data buffering and synchronization, a variety of slow lighting devices searched as practical delay system for practical precision. Basic solitons system is shown in Figure 2.

An optical modulator used for conversion an NRZ to RZ. A difference frequency generator laser is employed to generate RZ pulse. Mach-Zehnder modulator is employed for the modulation of the NRZ data transmission then several NRZ signals are multiplexed before the conversion in RZ pulse. At the receiving end it requires to convert from RZ to NRZ. NRZ signal then de-multiplexed for specific NRZ data for each channel. 


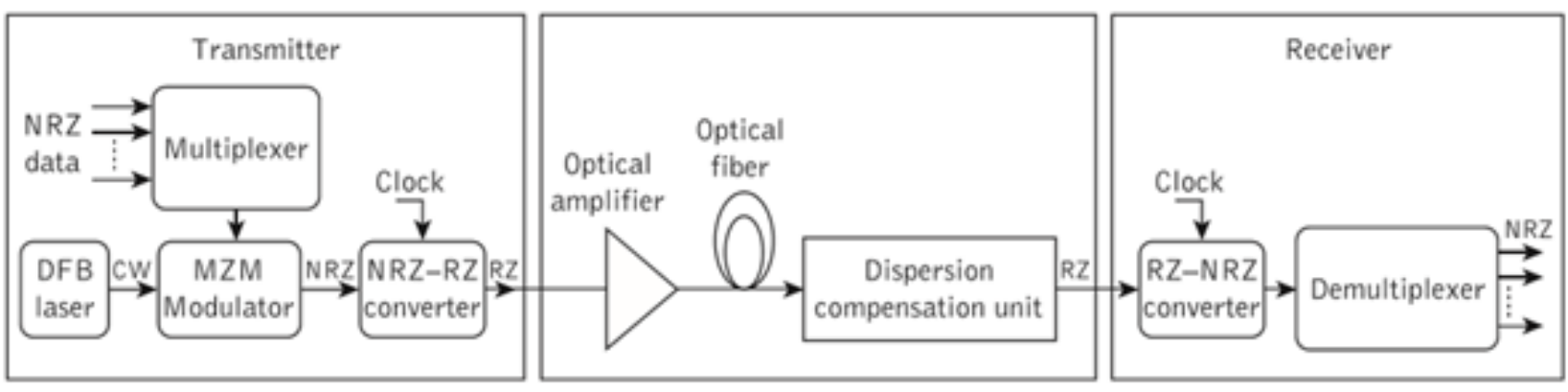

Fig 2. Block diagram for solitons transmission system in optical fiber.

Solitons pulse is better than NRZ pulse in the following way

1. It is important to obtain the generation of optical solitons pulse where transmitter can produce ultrafast RZ pulse. Mode lock fiber ring laser can be used to accomplish RZ pulse ${ }^{(13)}$

2. Solitons can be generated at a loss minimum area around 1550nm.

3. Time and polarized multiplexed method can be used for a long-distance transmission.

4. It is useful for long distance communication as there is no wave distortion over large length.

5. Solitons are free with elastic nature from dispersion and collision. The amplitude and frequency of solitons does not change after the collision but position and phase may change.

6. Solitons with two counter propagating passes without affecting each other, main difference between them is encoded digital with solitons and with NRZ ${ }^{(14)}$. NRZ and solitons pulse is shown in Figure 3.

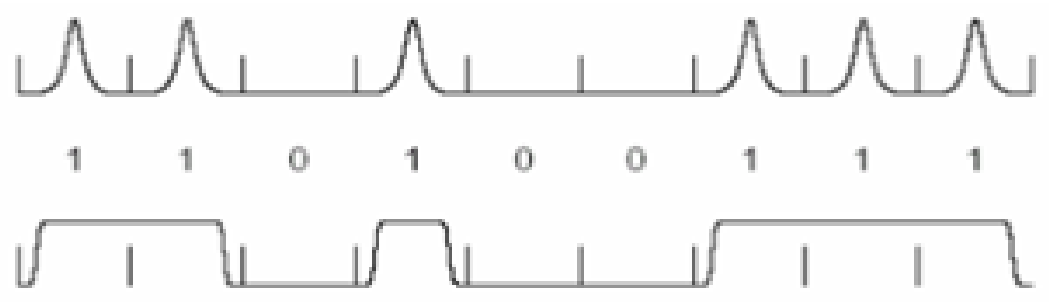

Fig 3. NRZ and solitons pulse

Solitons pulse propagate at a long range. Amplitude and timing are preserved time to time as pulse propagates without any change within bit parameters. A pulse propagates without producing any energy due to proper balance of Kerr linearity and $2^{\text {nd }}$ order non linearity dispersion. Some physical process may eliminate these balances resulting in detect of bit pattern when many solitons pulses are propagated closely with each other. It is necessary to keep reliable distance between two successive solitons pulse to avoid interference. The dispersion on the fiber is handled by optical solitons. It needs to take amplifier in dispersion management scheme if distance between two amplifiers is high. The transmission (bit) rate of a solitons pulse depends on width of pulse $\tau$ and time period of bit $T_{0}$. Hence the maximum permissible transmission rate for soliton can be given as:

$$
\begin{gathered}
B_{T}=\frac{1}{T_{0}}=\frac{1}{2 q_{0} \tau} \\
q_{0}=\frac{1}{2}\left(\frac{T_{0}}{\tau}\right)
\end{gathered}
$$


Solitons width is characterized by second order dispersion coefficient $\beta_{2}$ and dispersion length $L_{D}$. Solitons pulse width is spread significantly due to dispersion effect.

$$
L_{D}=\frac{\tau^{2}}{\left|\beta_{2}\right|}
$$

Therefore,

$$
\tau=\sqrt{\left|\beta_{2}\right| L_{D}}
$$

Assuming solitons spread situation where optical amplifier are separated forty to fifty kilometers such as amplifier spacing $L_{A}$ is short from overall dispersion length

$$
\tau \gg \sqrt{\left|\beta_{2}\right| L_{A}}
$$

When $\left(L_{A} \ll L_{D}\right)$, the transmission rate for solitons is

$$
B_{T} \gg \frac{1}{2 q_{0}} \frac{1}{\sqrt{\left|\beta_{2}\right| L_{A}}}
$$

For a specific optical amplifier, spacing between 40 to $50 \mathrm{Km}$ with transmission rate for optical solitons given as $20 \mathrm{Gbit} / \mathrm{s}$

\section{Type of solitons}

\subsection{Spatial optical solitons}

Spatial self-trapping was provided by 1974 Bjorkholm and Ashkin in bulk vapor of sodium atoms. First observation of true solitons in CS2 gas took 11 years further studies Kerr solitons see in glass semiconductor polymers. Finally,bulk spatial solitons was seen in polymer cubic quantic nonlinearity. Spatial solitons study made rapid progress in mid 1990s when two new solitons supporting non-linear optical media ${ }^{(15)}$.

In quadratic media it was recognized as solitons generation 1970 which was working G-Stegeman group effective Kerr coefficient in quadratic medium. An effective Kerr media Jeman was first built and measured by Valter Di Salvo, Harald Tschan and Norbert Bachl.

Second Segev E, Halachmi N, Salzberg A, and Ben-Arie N. estimated that photorefractive material may support suitable soliton propagation. Later different types of photorefractive solitons were detected. Vortex solitons dynamics examined theoretically in many term and angular momentum spatial dynamics studied. A spatial dynamic has been studied in defining experimentally non photo refractive and quadratic no linear media.

\subsection{Temporal optical solitons}

In single mode, temporal optical solitons were dedicated in 1973 by Hasegawa an Tappert and first viewed as a change by Mollenauer in 1980. However, it was observed that when the pulse sufficiently high order dispersion and high order non-linear effect such as SRS and SS, they destroy solitons. In the dynamic analysis, solitons play a very important role especially for micro-structured optical fiber due to super component role. Temporal solitons are further classified as:

\subsubsection{Bright temporal solitons}

In order to discuss solitons in a general setting we drive the dimension NLSE ${ }^{(16)}$ by introducing the standard non-dimensional variable

$$
\widetilde{z}=\frac{z}{2 k_{0} r_{0}^{2}}, \quad \widetilde{x}=\frac{x}{r_{0}}, \quad \widetilde{A}=\frac{n_{0}}{k_{0} r_{0}} A
$$

Where, $r_{0}$ is the beam width

$$
i A_{z}(z, \vec{x})+\nabla^{2} A+\Delta n\left(|A|^{2}\right) \mathrm{A}=0
$$

where, tildes dropped for simplicity perception to find equation of solitons we seek nonlinear bound state of form's equation

$$
A(z, \vec{x})=e^{i v z} u(\vec{x} ; v)
$$


Where, $u$ is a real function so the equation for the solitons profile is

$$
\nabla^{2} u(\vec{x} ; v)+\triangle n\left(|u|^{2}\right) u-v u=0
$$

Parameter $v$ is sometimes called as propagation constant or the solitons Eigenvalue (term originates from the linear Schrodinger equations. It describes the accumulated phase due to linear effect or equivalent change of linear propagation constant $\left(k_{0}=\vec{k} \cdot \widetilde{z}\right)$

Because solitons amplitude is radically symmetric function, the boundary condition for solitons equation.

$$
u^{\prime}(|\vec{x}|)=0, u(\infty)=0
$$

\subsubsection{Dark temporal solitons}

In Dark solitons, a localized pulse that appears with fast intensified on constant wave environment not like bright complement on a zero-intensity background. Dark solitons perform significant stability beside noise and fiber loss. It is used for signal processing, communication and switching because of its inherent stability ${ }^{(17)}$

\subsubsection{Optical spatio-temporal solitons}

Solitons is likely to generate a pulse transverse in localized dimension of space as well in time. This type of solitons (3+1) dimension NLSE is called spatiotemporal solitons. The experiment towards the production has been slow in its broad study by spatial Temporal solitons $(1+1) \mathrm{D},(2+1) \mathrm{D}$ and $(3+1) \mathrm{D}$. One of the reasons that why the power of the formation of stable spato-temporal was not yet theoretically identifiable. Evidence for spatio-temporal were given by an intelligent group by the cubic media and the brand group in cubic media, although in these cases beam splits in space and time after propagating a few characteristics length ${ }^{(18)}$

\subsection{Optical vector solitons}

Vector solitons refers to both of those solitons which have mutually added component. Individual solitons are twisted weakly birefringent single mode fiber where the light transmission is mathematically coded by the NLSE. Various vector solitons dark -dark vector, dark-bright vector and bright-bright-vector solitons ${ }^{(19)}$

\subsubsection{Dark-Dark vector solitons}

It appears in terms of defocusing Manakov model and assorted with focusing and defocusing non-linearity.

$$
\begin{gathered}
i u_{z}-u_{t t}+2 \mu\left(|u|^{2}+\left(\left.v\right|^{2}\right) u=0\right. \\
i v_{z}-u_{t t}+2 \mu\left(|u|^{2}+|v|^{2}\right) v=0 \\
u(z, t)=-\frac{\tau_{1}}{2}\left[\left(1+Z_{g}\right)-\left(1-Z_{g}\right) \tanh \left(\frac{p_{1} t-\Omega_{1} z+\xi_{1}^{0}}{2}\right)\right] \times e^{i\left[I_{1} t-\left(\lambda-I_{1}^{2}\right) z+\psi_{1}^{0}\right]} \\
v(z, t)=-\frac{\tau_{2}}{2}\left[\left(1+Z_{h}\right)-\left(1-Z_{h}\right) \tanh \left(\frac{p_{2} t-\Omega_{2} z+\xi_{2}^{0}}{2}\right)\right] \times e^{i\left[I_{1} t-\left(\lambda-I_{1}^{2}\right) z+\psi_{1}^{0}\right]}
\end{gathered}
$$

Where $I_{1} I_{2}, \psi_{1}^{0}, \psi_{2}^{0}, P_{1}, \Omega_{1}$, and $\xi_{1}^{0}$ are all real constant, $\tau_{1}, \tau_{2}$ and $Z_{h}$ are all complex constants, and $\left|Z_{g}\right|=\left|Z_{g}\right|=1$

\subsubsection{Dark bright-vector solitons}

Dark Bright solitons was investigated the NLSEs

$$
i \frac{\partial \vec{e}}{\partial z}+\frac{1}{2} \frac{\partial^{2} \vec{e}}{\partial t^{2}}-|\vec{e}|^{2} \vec{e}=\overrightarrow{0}
$$


Where $z$ is distance and $t$ is time coordinate and $\vec{e}=\left(e_{+}, e_{-}\right)^{T}$ represents transversely polarized light. In Hirota's bilinear method, dark-bright vector solitons given as ${ }^{(20)}$

$$
\begin{gathered}
e_{+}=\tau\{i \sin \varnothing+\cos \varnothing \tanh [a(t-b z)]\} e^{i c t+i\left[\frac{c^{2}}{2}+\tau^{2}\right] z,} \\
e_{-}=\sqrt{\tau^{2} \cos ^{2} \varnothing-a^{2}} \operatorname{sech}[a(t-b z)] e^{i b t+i\left[\frac{b^{2}-a^{2}}{2}-\tau^{2}\right] z}
\end{gathered}
$$

Where $a, b, c$ and $\tau$ are constant satisfying $a^{2}+\left(c^{2}-b^{2}\right) \leq \tau^{2}$ and $\varnothing=\arctan \frac{c-b}{a}$

\subsubsection{Bright-bright-vector solitons}

Two polarity component bright-bright solitons mention the solitons containing solitons bright pulse. The Manakov system ${ }^{(21)}$

$$
\begin{aligned}
& i u_{t}+u_{x x}+\left(|u|^{2}+|v|^{2}\right) u=0 \\
& i v_{t}+v_{x x}+\left(|u|^{2}+|v|^{2}\right) v=0
\end{aligned}
$$

And the bright-bright solitons solution as

$$
\begin{aligned}
(u, v)^{T}(x, t) & =2 \eta \operatorname{sech}\left[2 \eta\left(x+4 \xi t-x_{0}\right)\right] e^{-2 i \xi x-4 i\left(\xi^{2}-\eta^{2}\right) t} \\
& \times \frac{1}{\sqrt{\left|\propto_{1}\right|^{2}+\left|\propto_{2}\right|^{2}}}\left(\propto_{1}, \propto_{2}\right)^{T}
\end{aligned}
$$

$\xi$ and $\eta$ are the parameters decide the velocity and amplitude $x_{0}$ is the coordinate at $t=0$, and $\alpha_{1}, \alpha_{2}$ are two complex variables.

\section{Advantages of optical solitons}

\subsection{Solitons as trains of high bitrate optical fiber communication}

A binial frequency input signal is transferred through the fiber with active elaboration. An advanced spectral component is produced during non-linear multiplication through the fiber and simultaneously, phase or amplitude of the ingredients are autoadjusted so that an enhanced quality solitons train is generated. Solitons tenure is dogged by the total effective amplification and input signal ${ }^{(22)}$

\subsection{Memory generations}

Optical system of micro ring resonator is presented to create femto-second (fs) memory time of optical solitons. Solitons pulse are in the form of a bright solitons ${ }^{(23)}$ and inserted into micro ring resonator, then Micro ring resonator generate a highly chaotic signal and sliced it into ultra-short single and multi-solitons capacity of optical solitons pulse in pico and femto-seconds can be execute using the projected method. Systematic ultra-fast optical solitons can be used to create optical quantum memory ${ }^{(24)}$

\subsection{Improvement of microwave fiber optic link efficiency}

Stimulated Brillouin Scattering can be used in a self- regulating, self-tuning carrier filter to enhance the modulation depth and efficiency of fiber optic links. Improvements of $8 \mathrm{~dB}$ have been achieved when light is injected into a single core fiber which is eight times higher than the SBS threshold ${ }^{(25)}$

\subsection{Increase the capacity of WDM optical system}

The WDM system dependent on solitons is superior to CW pulse system as the power modulation in earlier system was 9.79015 $\mathrm{dB}$ and the posterior system had $8.4250 \mathrm{~dB}$. WDM ${ }^{(26)}$ performance of solitons dependent optical system provides enhanced result comparable to CW system. Reduced jitter bit error rate and reduced signal power improves the communication system. Solitary approach to the entire concept made transmission system stable ${ }^{(27)}$ 


\section{Application of optical solitons}

\subsection{Solitons Amplification}

When pulse propagate through the fiber, due to absorption of energy there is some loss of energy. Such losses disrupt some communication. These fiber losses are solitons extend optical communication especially apart from various other applications. These losses are compensating by optical pulses. There are two ways for solitons promotion called lumped and distributed amplification ${ }^{(28)}$. Lumped scheme ${ }^{(29)}$ optical amplifier provides a short range of amplification. Distribution amplifier is one which the transmission fiber is utilized as gain medium by multiplexing a pump wavelength with signal wavelength. Distribution amplification is done by stimulated Raman scattering ${ }^{(30)}$ and $\operatorname{EDFA}^{(31,32)}$.

\subsection{Pulse compression}

Solitons compression is a technique for compression of ultra-short pulse in a fiber. Fundamentally solitons pulse energy and the pulse duration is proportional to the group velocity dispersion divided by the nonlinearity of the fiber. Thus, solitons duration can be reduced if dispersion is reduced while keeping constant the pulse duration. Significantly pulse compression can be obtained by propagating the pulse through a dispersion decreasing fiber.

\subsection{Enhancement the bit rate}

Techniques for ultrafast optical signal processing expected to play major role in future 10-100 Gbit/s. A device which shows big efficiency in this field is wavelength nonlinear optical loop mirror which can perform wavelength conversion TDM and switching. For this a NRZ pulse required because it has great timing tolerance and minimum bandwidth required. WDM system change NRZ to RZ modulation ${ }^{(33)}$. Binary NRZ transmission easy to understand 0 is a low level and the 1 is high level. RZ is self-clocking but it requires more bandwidth. Both the system use kerr-linearity which affects the performance and distorting the signal at high speed and long distance ${ }^{(34)}$. WDM system increases the power without introducing the non-linearity. Non return to zero coding scheme positive voltage bit 1 and zero voltage defines bit 0 . Signal does not return to zero at the middle of the bit. NRZ codes are easy to engineer and it makes efficient use of bandwidth. However, dispersion managed solitons in advance optics technology play a important role in long distance network.

\subsection{Reshaping of optical signal in glass fiber}

Optical solitons is a self-reinforcing solitary wave packet that maintains its shape while it propagates at constant speed without distortion in optical fiber. However, the pulse width increases due to fiber loss. So, a periodic amplification is needed to retain the original shape at a long distance and it is determined by the group velocity dispersion (GVD). The shape of a solitons does not change practically even after thousands of kilo meter of repeated amplification ${ }^{(35)}$.

\section{Conclusion}

The study analysises the $4^{\text {th }}$ generation fiber optic communication system where visual amplification is applied in order to decrease the demand for repeaters along with WDM to increase the information carrying capacity. Similarly, the futuristic work going on in the $5^{\text {th }}$ phase of Optical Fiber Communication for expending the distances has also been discussed. The future lies in the low loss window promising extension of range to 1.30 to $1.65 \mathrm{um}$. It seems to be a promising development that includes the idea of visual solitons pulses that maintain their body by counteracting. That results in distribution with nonlinear effects of this material by applying pulses of specific shape. All the major aspects of Solitons including, types, working principle, applications and futuristic uses have been analyzed in details. It will help to undertake further research work in this innovative area.

\section{References}

1) Sreedevinai S, Prem V. Solitons A Promising Technology in Optical Communication. International Journal of Science and Research. 2014. Available from: httpsdoi.org/10.1088/0305-4470/33/18/308.

2) Chawda DNB. Soliton and its underlaying principle of information carrying bits. International Journal of Conceptions on computing and Information Technology. 2016;5. Available from: https:/www.doi.org/10.36106/paripex.

3) Song Y, Wang Z, Wang C, Panajotov K, Zhang H. Recent progress on optical rogue waves in fiber lasers: status, challenges, and perspectives. Advanced Photonics. 2020;2:1-1. Available from: https://dx.doi.org/10.1117/1.ap.2.2.024001. 
4) Song YF, Zhang H, Zhao LM, Shen DY, Tang DY. Coexistence and interaction of vector and bound vector solitons in a dispersion-managed fiber laser mode locked by graphene. Optics Express. 2016;24(2):1814-1814. Available from: https://dx.doi.org/10.1364/oe.24.001814.

5) Wang Z, Wang X, Song Y, Liu J, Zhang H. Generation and pulsating behaviors of loosely bound solitons in a passively mode-locked fiber laser. Physical Review A. 2020;101(1). Available from: https://dx.doi.org/10.1103/physreva.101.013825.

6) Mao D, Jiang B, Gan X, Ma C, Chen Y, Zhao C, et al. Soliton fiber laser mode locked with two types of film-based Bi_2Te_3 saturable absorbers. Photonics Research. 2015;3(2):A43-A43. Available from: https://dx.doi.org/10.1364/prj.3.000a43.

7) Guo B, Wang SH, Wu ZX, Wang ZX, Wang DH, Huang H, et al. Sub-200 fs soliton mode-locked fiber laser based on bismuthene saturable absorber. Optics Express. 2018;26(18):22750-22750. Available from: https://dx.doi.org/10.1364/oe.26.022750.

8) Wang Q, Chen Y, Miao L, Jiang G, Chen S, Liu J, et al. Wide spectral and wavelength-tunable dissipative soliton fiber laser with topological insulator nano-sheets self-assembly films sandwiched by PMMA polymer. Optics Express. 2015;23(6):7681-7681. Available from: https://dx.doi.org/10.1364/oe.23. 007681.

9) Tariq KUH, Seadawy AR. Bistable Bright-Dark solitary wave solutions of the $(3+1)$-dimensional Breaking soliton, Boussinesq equation with dual dispersion and modified Korteweg-de Vries-Kadomtsev-Petviashvili equations and their applications. Results in physics. 2017;7. Available from: https://doi.org/10.1016/j.rinp.2017.03.001.

10) Wazwaz AM. A variety of optical solitons for nonlinear Schrödinger equation with detuning term by the variational iteration method. Optik International Journal for Light and Electron Optics. 2019;196. Available from: https://doi.org/10.1016/j.ijleo.2019.163169.

11) Kruglov VI, Harvey JD. Solitary waves in optical fibers governed by higher-order dispersion. Physical Review. 2018. Available from: https://doi.org/10. 1103/PhysRevA.98.063811.

12) Aslan EC. Optical soliton solutions of the NLSE with quadratic-cubic-Hamiltonian perturbations and modulation instability analysis. Optik. $2019 ; 196$. Available from: https://dx.doi.org/10.1016/j.ijleo.2019.04.008.

13) Bowen P, Erkintalo M, Neil GR, Broderick. Large net-normal dispersion Er-doped fiber laser mode-locked with a nonlinear amplifying loop mirror. Optics Communication. 2018. Available from: https://doi.org/10.1016/j.optcom.2017.10.053.

14) Yang X, Shen L, Ramamurthy B. Survivable lightpath provisioning in WDM mesh networks under shared path protection and signal quality constraints. Journal of Lightwave Technology. 2005;23(4):1556-1567. Available from: https://dx.doi.org/10.1109/jlt.2005.844495.

15) Navarrete A, Paredes A, Salgueiro JR, Michinel H. Spatial solitons in thermo-optical media from the nonlinear Schrödinger-Poisson equation and darkmatter analogs. Physical Review A. 2017;95(1):13844-13844. Available from: https://dx.doi.org/10.1103/physreva.95.013844.

16) Wu YT, Hsiao SC. Generation of stable and accurate solitary waves in a viscous numerical wave tank. Ocean Engineering. 2018;167:102-113. Available from: https://dx.doi.org/10.1016/j.oceaneng.2018.08.043.

17) Chen Z, Segev M, Christodoulides DN. Optical spatial solitons: historical overview and recent advances. Reports on Progress in Physics. 2012;75(8):086401086401. Available from: https://dx.doi.org/10.1088/0034-4885/75/8/086401.

18) Sazonov VS. Analytical description of the propagation of spatiotemporal solitons in fibers. Physical Review A. 2019;100(4):43828-43828. Available from: https://dx.doi.org/10.1103/physreva.100.043828.

19) Wang BH, Lu PH, Dai CQ, Chen YX. Vector optical soliton and periodic solutions of a coupled fractional nonlinear Schrödinger equation. Results in Physics. 2020;17:103036-103036. Available from: https://dx.doi.org/10.1016/j.rinp.2020.103036.

20) Song Y, Shi X, Wu C, Tang D, Zhang H. Recent progress of study on optical soliton in fiber laser. Applied Physics. 2019. Available from: https: //doi.org/10.1063/1.5091811.

21) Yakupyildırım. Optical soliton molecules of Manakov model by modified simple equation technique. Optik- International Journal for Light and Electron. 2019;185. Available from: https://doi.org/10.1016/j.ijleo.2019.04.046.

22) Mamyshev PV, Chernikov SV, Dianov EM. Generation of fundamental soliton trains for high-bit-rate optical fiber communication lines. IEEE Journal of Quantum Electronics. 1991;27(10):2347-2355. Available from: https://dx.doi.org/10.1109/3.97280.

23) Zhao Y, Chen Y, Dai J, Wang Y, Wang W. Bright Soliton Solution of (1+1)-Dimensional Quantum System with Power-Law Dependent Nonlinearity. Advances in Mathematical Physics. 2019;2019:1-5. Available from: https://dx.doi.org/10.1155/2019/8264848.

24) Amiriand S, Ali J. Femtosecond Optical Quantum Memory Generation Using Optical Bright Soliton. Journal of Computational and Theoretical Nanoscience. 1480;11:1480-1485. Available from: https://doi.org/10.1166/jctn.2014.3521.

25) Williams KJ, Esman RD. Stimulated Brillouin scattering for improvement of microwave fibre-optic link efficiency. Electronics Letters. 1994;30(23):19651966. Available from: https://dx.doi.org/10.1049/el:19941344.

26) Singh M, Saini HS. High Performance Soliton WDM Optical Communication System. IEEE Computer society. 2014. Available from: https:/doi.org/10. 1109/ICACC.2014.11,2014.

27) Karaki J, Pincemin E, Grot D, Guiliossou T, Jaouen Y, Bidan RL, et al. Dual-polarization multi-band OFDM versus single-carrier DP-QPSK for 100 Gbps long-haul WDM transmission over legacy infrastructure. In: and others, editor. 38th European Conference and Exhibition on Optical Communications. Amsterdam, Netherlands. 2014. Available from: https://doi.org/10.1364/ECEOC.2012.P4.17.

28) Liao Z, Agrawal G. Role of Distributed Amplification in Designing High-Capacity Soliton Systems. Optics Express. 2001;9(2):66-66. Available from: https://dx.doi.org/10.1364/oe.9.000066.

29) Poutrina E, Agrawal GP. Timing jitter in dispersion-managed soliton systems with distributed, lumped, and hybrid amplification. Journal of Lightwave Technology. 2002;20(5):790-797. Available from: https://dx.doi.org/10.1109/jlt.2002.1007931.

30) Sirleto L, Ferrara MA. Fiber Amplifiers and Fiber Lasers Based on Stimulated Raman Scattering: A Review. Micromachines. 2020;11:247-247. Available from: https://dx.doi.org/10.3390/mil1030247.

31) Bowen P, Erkintalo M, Broderick GRN. arge net-normal dispersion Er-doped fiber laser mode-locked with a nonlinear amplifying loop mirror. Optics Communication. 2018. Available from: https://doi.org/10.1016/j.optcom.2017.10.053.

32) Subramanian K, Alagesan T, Mahalingam A, Rajan MSM. Propagation properties of optical soliton in an erbium-doped tapered parabolic index nonlinear fiber: soliton control. Nonlinear Dynamics. 2017;87(3):1575-1587. Available from: https://dx.doi.org/10.1007/s11071-016-3134-1.

33) Badraoui N, Berceli T. Modeling and design of soliton propagation in WDM optical systems. In: and others, editor. International Conference on Optical Network Design and Modeling (ONDM), Budapest. 2017;p. 1-6. Available from: https://doi.org/10.23919/ONDM.2017.7958524.

34) Biswas A, Ekici M, Sonmezoglu A, Belic RM. Highly dispersive optical solitons with Kerr law nonlinearity by exp-function. Optik. 2019;185:121-125. Available from: https://dx.doi.org/10.1016/j.ijleo.2019.03.069.

35) Sirleto L, Ferrara MA. Fiber Amplifiers and Fiber Lasers Based on Stimulated Raman Scattering A Review. Micromachines. 2020. Available from: https://doi.org/10.3390/mi11030247. 